\section{Effects of strenuous exercise on haemostasis}

\section{J E Smith}

See end of article for author's affiliation

.....................

Correspondence to: Dr Smith, 4 Fort Terrace, Plymouth PL6 5BU, UK: jason.smith75@virgin.net

Accepted

20 October 2002

\begin{abstract}
Objectives: To review the effects of exercise on haemostasis and examine the possible clinical sequelae of these changes.

Methods: The search strategy included articles from 1966 to August 2002 using Medline and SportDiscus databases, and cross referencing the bibliographies of relevant papers.

Results: Exercise results in activation of both the coagulation and fibrinolytic cascades, as shown by a reduction in whole blood clotting time and activated partial thromboplastin time, an increase in the activity of several components of the cascades, and an increase in fibrin degradation products. In vitro tests suggest that coagulation remains activated after fibrinolysis has returned to baseline levels.

Conclusions: Both the coagulation and fibrinolytic cascades are stimulated by strenuous exercise, but the temporal relation between the two and its clinical significance remains to be clarified. Doctors and athletes should be aware of the haemostatic changes induced by exercise, and further work is needed to clarify the possible role of these changes in sudden cardiac death.
\end{abstract}

$\mathrm{H}$ aemostasis is achieved through a delicate equilibrium between the coagulation and fibrinolytic cascades. All the components of these cascades exist in the circulation as inactive proteins, which are converted into their active enzymatic form when the cascades are activated. The intrinsic and extrinsic pathways merge to produce thrombin from prothrombin, which in turn stimulates the production of fibrin from fibrinogen. When fibrin becomes cross linked and combines with platelets, a clot is formed. This process is regulated by inhibitory mechanisms, including fibrinolysis, the process by which fibrin is broken down into soluble components. Abnormalities of haemostasis are implicated in the pathogenesis of several diseases, and many therapeutic processes alter the balance of haemostatic control.

Regular exercise is generally associated with favourable alterations in risk from cardiovascular morbidity and morbidity, but strenuous exercise has been implicated in the pathogenesis of sudden death. ${ }^{12}$ The mechanism behind this is not clear. Exercise has been shown to affect both coagulation and fibrinolysis, and the relation between the activation of the two cascades has implications in patients at risk of developing intravascular thrombus. If exercise preferentially activates fibrinolysis, then it may be of benefit in these subjects, but if coagulation is preferentially activated, it may potentiate devastating occlusion of a coronary or cerebral vessel. The purpose of this article is to provide a summary of the evidence of the effects of exercise on haemostasis and to examine the potential implications of the findings.

\section{METHOD}

The database was obtained by a computerised search of Medline and SportDiscus from 1966 to August 2002, and cross referencing the bibliographies of articles found. Keywords used in the search were exercise, blood coagulation, blood coagulation factors, and fibrinolysis.

\section{FINDINGS \\ Coagulation}

It has been known for many years that blood taken immediately after exercise is hypercoagulable, most easily demonstrated by testing whole blood clotting time. ${ }^{3-5}$ This is also reflected by a change in activated partial thromboplastin time, which measures the activity of the intrinsic and common pathways, after exercise such that it is reduced by $7-38 \% .^{6-18}$
Studies of the effect of exercise on prothrombin time (a measure of the activity of extrinsic and common pathways) and thrombin time (a measure of common pathway activity) have given conflicting results. Most show no demonstrable effect on prothrombin time, ${ }^{19-22}$ although some have shown a significant shortening of thrombin time..$^{12} 19$

Changes in individual components of the coagulation cascade as a result of exercise have also been shown. Factor VIII activity has been shown to increase by 200$400 \%,{ }^{4} 9_{11131723-31}$ and the degree of increase in factor VIII activity is dependent on volume and intensity of exercise. ${ }^{32} 33$ Studies of fibrinogen level have given conflicting results, with some showing no difference after exercise, ${ }^{19} 263435$ some showing an increase, ${ }^{913}$ and some showing a decrease. ${ }^{14}{ }^{36}$ A recent study has postulated that fibrinogen levels are decreased after maximal and submaximal exercise, suggesting consumption during the coagulation cascade, ${ }^{37}$ but this is only evident when the reduction in plasma volume after exercise is taken into account. There is little convincing evidence of alterations in other components of the coagulation cascade. ${ }^{18}$

The cascade also has its own regulatory proteins that inhibit coagulation at various stages, such as antithrombin III and protein $\mathrm{C}$. These inhibitors of coagulation are also affected by exercise, although the results of studies looking at this are not so conclusive. Antithrombin III has been shown to fall in one study, ${ }^{38}$ although little change was seen in others. ${ }^{13}{ }^{14}$ Ferguson et $a l^{12}$ describe a rise in absolute antithrombin III concentration, but a fall in concentration once corrections were made for plasma volume, suggesting consumption of the factors. In two studies, the level of thrombin-antithrombin III complexes rose significantly with exercise, ${ }^{14}{ }^{34}$ suggesting that there had been in vivo formation of thrombin that had been neutralised by the antithrombin III. This was confirmed in later studies which found that thrombin-antithrombin III complexes increased after maximal exercise, and this was more pronounced after prolonged exercise. ${ }^{15} 1639$

\section{Fibrinolysis}

Fibrinolysis is the process by which fibrin is broken down and made soluble. It is stimulated by plasmin, which is derived from its inactive precursor, plasminogen, as a result of the action of plasminogen activating factors. The most important of these is tissue-type plasminogen activator (t-PA). As a result of fibrinolysis, fibrin degradation products are produced. Plasminogen activator inhibitor (PAI-1) inhibits the action of t-PA, 
and plasmin is inhibited by antithrombin III. Fibrinolysis can be measured in various ways, the most common being a measurement of the time taken for the test sample to break down a standardised clot, as shown by the euglobulin lysis time.

Fibrinolysis is activated in response to exercise. This is usually attributed to an increase in t-PA, which is released by the vascular endothelium. ${ }^{40}{ }^{41}$ Significant rises in t-PA have been found in various exercise protocols. ${ }^{13} 16172231394243$ This increase is positively related to exercise intensity and duration, such that the higher the intensity and duration of exercise, the more exaggerated the effect. ${ }^{2033} 3544-46$ There is also some evidence that aerobic training enhances the activation of fibrinolysis after exercise. ${ }^{42}$

Fibrinolysis is controlled not only by the activators, but also by inhibitors such as PAI-1, another substance released by damaged endothelial cells. Circulating t-PA is either in its active form or, when combined with PAI-1, it is inactivated. The activity of PAI- 1 can be measured (by combining the test sample with a standard solution containing one chain t-PA, and measuring residual t-PA level after the reaction), and has been shown to fall after both endurance and resistance exercise..$^{15} 1955^{43}$ Rocker et al ${ }^{22}$ found that PAI-l activity was undetectable after a marathon, suggesting that it had all been consumed in bound form with plasminogen activator.

The end products of fibrinolysis, fibrin degradation products, have been shown to increase after endurance exercise of different types. ${ }^{131416223942}$ D-dimers, which are products of the breakdown of activated factor XIII (fibrin stabilising factor) and fibrin, are often used as a marker of fibrin degradation, and have also been shown to increase. ${ }^{131517202239}$ This would suggest that the activation of fibrinolysis is not simply an in vitro response to exercise, but does actually occur in vivo. ${ }^{41}$ It has been suggested that subjects have a fibrinolytic capacity relating to their risk of developing thromboembolic or atherosclerotic disease, and this capacity is impaired in patients with some forms of illness. ${ }^{41}$

\section{Platelet aggregation}

As a result of vessel wall injury, substances that activate platelets are exposed, such as collagen. Platelets adhere to these and become activated, releasing substances that promote platelet adherence and vasoconstriction, such as thromboxane $\mathrm{A}_{2}$ and $\mathrm{ADP}$, as well as platelet specific proteins such as $\beta$-thromboglobulin and platelet factor 4. Counteracting substances such as prostacyclin (which acts as a vasodilator) regulate this process. Platelet activation can be estimated in several ways, one of which being estimation of the level of platelet specific proteins such as platelet factor 4 or $\beta$-thromboglobulin, which are both in vivo indicators of platelet activation and degranulation.

The platelet count has repeatedly been shown to rise after acute exercise..$^{24047}$ This is presumed to be due to mobilisation of platelets from the spleen, bone marrow, and other reticuloendothelial organs, in response to stimulation by catecholamines released during exercise. ${ }^{48}$ However, the effect of exercise on platelet function is less clear cut, with some reports of increased aggregation, and some of decreased aggregation. ${ }^{41} \beta$-Thromboglobulin was found to be increased after graded exercise, with higher values after more strenuous exercise ${ }^{16}$ and in less trained individuals ${ }^{48}$ Both platelet factor 4 and $\beta$-thromboglobulin were found to be increased after a marathon. $^{2130}$ However, in one marathon study, despite increased levels of $\beta$-thromboglobulin, platelet aggregation was found to be decreased after exercise, ${ }^{30}$ suggesting that the platelets had been activated, had released $\beta$-thromboglobulin, and then reverted to a less reactive state. The mechanisms behind platelet activation during exercise are not fully understood, but may be related to shear stress causing endothelial damage, increases in plasma catecholamines, thrombin generation, and mobilisation of more active platelets from the reticuloendothelial system. ${ }^{21}$
Table 1 Summary of effects of exercise on markers of clotting and fibrinolysis

\begin{tabular}{ll}
\hline Parameter & Effect of exercise \\
\hline Clotting & Decreased \\
APTT & Unchanged \\
PT & Decreased \\
Thrombin time & Increased \\
Factor VIII activity & \\
Fibrinolysis & Increased \\
t-PA (activation) & Decreased \\
PAl-1 (inhibition) & \\
Platelet aggregation & Increased \\
Platelet count & Uncertain \\
Aggregation & \\
\hline
\end{tabular}

APTT, Activated partial thromboplastin time; PT, prothrombin time; t-PA, tissue-type plasminogen activator; PAl-1, plasminogen activator inhibitor.

\section{DISCUSSION}

The findings of this report support the hypothesis that both coagulation and fibrinolysis are stimulated by strenuous exercise, and these effects are summarised in table 1. The activation of these systems is less pronounced in athletes than untrained runners. ${ }^{38}$ In healthy subjects, the activation of the cascades should work to maintain the balance between the two systems ${ }^{16}{ }^{38}{ }^{47}$ but if the activation of these systems is not in balance, a predisposition to thrombus formation may be the result. In a group of patients followed up for one hour after exercise, Hegde $e t$ al $^{17}$ found that, although t-PA antigen level declined gradually over the hour, activated partial thromboplastin time remained shortened, suggesting continued activation of coagulation. These findings were similar to those of Lin et al, ${ }^{31}$ who found that clotting activity (factor VIII activity) remained elevated for some hours, while fibrinolytic activity fell sharply. In a field study of marathon runners, Siegel et al ${ }^{49}$ found that markers of coagulation remained activated the day after the race, whereas fibrinolytic activity had returned to baseline. In theory, this could predispose some subjects to a dangerous tendency to form intravascular thrombus, although other effects of exercise such as vasodilatation and increased blood flow should work to counteract this. There is general agreement that exercise is beneficial to health, and has many positive effects on various aspects of physiology such as blood lipid profile, cardiovascular disease, bone health, and psychological wellbeing. However, how much exercise is good for which patients is a more difficult question. Very strenuous physical activity such as marathon running may not be beneficial to some people, and one of the mechanisms behind this may be an unequal activation of the coagulation and fibrinolytic cascades.

Many of the conclusions drawn from these studies have limitations. There is no standardised protocol for defining exercise intensity or duration, uniformity of sampling, and populations studied. Studies have varied from laboratory based treadmill protocols at a given percentage of maximum oxygen consumption to field based protocols in events as diverse as running, cycling, and triathlon. Subjects used for these studies have tended to be young and healthy, and so it is difficult to extrapolate their results to patients with abnormal or diseased cardiovascular systems, such as the elderly and those with atherosclerosis, or those with multiple risk factors for cardiac disease. It should be stressed that it is not clear how the changes in laboratory values of factors involved in coagulation and fibrinolysis relate to clinical disease processes, and many of the conclusions drawn in these studies are speculative.

Resistance exercise is promoted by the American College of Sports Medicine and the American Heart Association, especially in the older population, but studies into its effects on the balance of coagulation and fibrinolysis are limited. 


\section{Take home message}

Exercise has been shown to stimulate both coagulation and fibrinolysis, although whether this activation is balanced has yet to be clarified. If unbalanced, this could be responsible for adverse clinical events in susceptible people.

It would be of benefit to examine the temporal relation of alterations in the coagulation and fibrinolytic cascades in more detail, to look for further evidence of imbalance in the activation of these two systems. This should clarify whether suggestions that this may be the mechanism responsible for episodes of sudden death after exercise are justified. Opportunities to examine the effects of exercise on the clotting process using more sophisticated techniques such as thromboelastography, which gives a graphical representation of the clotting process and can accurately detect hypercoagulable states, ${ }^{50}$ may provide the solution to the debate.

\section{Conclusion}

Both the coagulation and fibrinolytic cascades are stimulated by strenuous exercise, but the temporal relation between the two and its clinical significance have yet to be clarified. Unfavourable haemostatic changes at the extremes of exercise intensity may predispose to the formation of intravascular thrombus and may contribute to the phenomenon of sudden cardiac death after exercise. Further work is suggested to investigate this possibility, especially in the older age group and those with coronary risk factors.

\section{Authors' affiliations}

J E Smith, Academic Department of Sports Medicine, Royal London Hospital (Mile End), Bancroft Road, London E I, UK

\section{REFERENCES}

1 Maron BJ, Poliac LC, Roberts WC. Risk of sudden cardiac death associated with marathon running. J Am Coll Cardiol 1996;28:428-31.

2 Albert CM, Mittleman MA, Chae CU, et al. Triggering of sudden death by vigorous exertion. N Engl J Med 2000;343:1355-61.

3 Ferguson EW, Guest MM. Exercise, physical conditioning, blood coagulation and fibrinolysis. Thromb Diasthes Haemorrh 1974;31:63-71

4 latridis SG, Ferguson JH. Effect of physical exercise on blood chemistry and clotting and fibrinolysis. J Appl Physiol 1963;18:337-44.

5 Ikkala E, Myllyla G, Sarajas HSS. Haemostatic changes associated with exercise. Nature 1963;199:459-61.

6 Berkada B, Akokan G, Derman U. Fibrinolytic response to physical exercise in males. Atherosclerosis 1971;13:85-91.

7 Collen D, Semeraro N, Tricot JP, et al. Turnover of fibrinogen, plasminogen, and prothrombin during exercise in man. J Appl Physiol 1977;42:865-73.

8 Mandalaki T, Dessypris A, Louizou C, et al. Marathon run. I. Effects on blood coagulation and fibrinolysis. Thromb Haemost 1977:37:444-55.

9 Ferguson EW, Barr CF, Bernier LL. Fibrinogenolysis and fibrinolysis with strenuous exercise. J Appl Physiol 1979;47:1 157-61.

10 Hyers TM, Martin BJ, Pratt DS, et al. Enhanced thrombin and plasmin activity with exercise in man. J Appl Physiol 1980;48:821-5.

11 Bartsch P, Schmidt EK, Straub PW. Fibrinopeptide A after strenuous physical activity at high altitude. J Appl Physiol 1982;53:40-3

12 Ferguson EW, Bernier LL, Banta GR, et al. Effects of exercise and conditioning on clotting and fibrinolytic activity in men. J Appl Physiol 1987;62:1416-21.

13 Arai $M$, Yorifuji $H$, Ikematsu $S$, et al. Influences of strenuous exercise (triathlon) on blood coagulation and fibrinolytic system. Thromb Res 1990;57:465-71.

14 Bartsch P, Haeberli A, Straub PW. Blood coagulation after long distance running: antithrombin III prevents fibrin formation. Thromb Haemost 1990;63:430-4.

15 Van den Burg PJ, Hospers JE, van Vliet M, et al. Changes in haemostatic factors and activation products after exercise in healthy subjects with different ages. Thromb Haemost 1995;74:1457-64

16 Weiss C, Seitel G, Bartsch P. Coagulation and fibrinolysis after moderate and very heavy exercise in healthy male subjects. Med Sci Sports Exerc 1998;30:246-51.
17 Hegde SS, Goldfarb AH, Hegde S. Clotting and fibrinolytic activity change during the $1 \mathrm{~h}$ after a submaximal run. Med Sci Sports Exerc $2001 ; 33: 887-92$

18 Bourey RE, Santoro SA. Interactions of exercise, coagulation, platelets and fibrinolysis: a brief review. Med Sci Sports Exerc 1988;20:439-46.

19 El-Sayed MS, Lin X, Ratty AJM. Blood coagulation and fibrinolysis at rest and in response to maximal exercise before and after a physical conditioning programme. Blood Coagul Fibrinolysis 1996;6:747-52.

20 Molz AB, Heyduck B, Lill $H$, et al. The effect of different exercise intensities on the fibrinolytic system. Eur J Appl Physiol 1993;67:298-304.

21 Rocker L, Drygas WK, Heyduck B. Blood platelet activation and increase in thrombin activity following a marathon race. Eur J Appl Physiol 1986;55:374-80.

22 Rocker L, Taenzer M, Drygas WK, et al. Effect of prolonged physical exercise on the fibrinolytic system. Eur J Appl Physiol 1990;60:478-81.

23 Cohen RJ, Epstein SE, Cohen LS, et al. Alterations of fibrinolysis and blood coagulation induced by exercise and the role of beta adrenergic receptor stimulation. Lancet 1968;i: 1264-6.

24 Hawkey CM, Britton BJ, Wood WG, et al. Changes in blood catecholamine levels and blood coagulation and fibrinolytic activity in response to graded exercise in man. Br J Haematol 1975;29:377-84.

25 Davis GL, Abildgaard CF, Bernaver EM, et al. Fibrinolytic and hemostatic changes during and after maximal exercise in males. J Appl Physiol 1976;40:287-92.

26 Collen D, Semeraro N, Tricot JP, et al. Turnover of fibrinogen plasminogen, and prothrombin during exercise in man. J Appl Physiol 1977;42:865-73

27 Brown JE, Baugh RF, Hougie C. Effect of exercise on the factor VIII complex: a correlation of the von Willebrand antigen and factor VIII coagulant antigen increase. Thromb Res 1979;15:61-7.

28 Wheeler ME, Davis GL, Gillespie WJ, et al. Physiological changes in hemostasis associated with acute exercise. J Appl Physiol 1986;60:986-90

29 Hansen JB, Wilsgard L, Olsen JO, et al. Formation and persistence of procoagulant and fibrinolytic activities in circulation after strenuous exercise. Thromb Haemos 1990;64:385-9.

30 Rock G, Tittley P, Pipe A. Coagulation factor changes following endurance exercise. Clin J Sport Med 1997;7:94-9.

31 Lin X, El-Sayed MS, Waterhouse J, et al. Activation and disturbance of blood haemostasis following strenuous physical exercise. Int J Sports Med 1999:20: 149-53.

32 El-Sayed MS. Fibrinolytic and hemostatic parameter response after resistance exercise. Med Sci Sports Exerc 1993;25:597-602.

33 Andrew M, Carter C, O'Brodovich H, et al. Increases in factor VIII complex and fibrinolytic activity are dependant on exercise intensity. $J$ Appl Physiol 1986;60;1917-22.

34 Herren T, Bartsch P, Haeberli A, et al. Increased thrombin-antithrombin III complexes after 1 hour of physical exercise. J Appl Physiol 1992;73:2499-504.

35 Rankinen T, Vaisanen S, Penttila I, et al. Acute dynamic exercise increases fibrinolytic activity. Thromb Haemos 1995;73:281-6.

36 Martin DG, Ferguson EW, Wigutoff $S$, et al. Blood viscosity responses to maximal exercise in endurance trained and sedentary female subjects. $J$ Appl Physiol 1985;59:348-53.

37 El-Sayed MS, Jones PG, Sale C. Exercise induces a change in plasma fibrinogen concentration: fact or fiction? Thromb Res 1999;96:467-72.

38 Mandalaki T, Dessypris A, Louizou C, et al. Marathon run. III. Effects on coagulation, fibrinolysis, platelet aggregation and serum cortisol levels. Thromb Haemost 1980;43:49-52.

39 Prisco D, Paniccia R, Bandinelli B, et al. Evaluation of clotting and fibrinolytic activation after protracted physical exercise. Thromb Res 1998;89:73-8

40 Marsh NA, Gaffney PJ. Some observations on the release of extrinsic and intrinsic plasminogen activators during exercise in man. Haemostasis 1980;9:238-47

41 El-Sayed MS. Effects of exercise on blood coagulation, fibrinolysis and platelet aggregation. Sports Med 1996;22:282-98.

42 De Paz JA, Lasierra J, Villa JG, et al. Changes in the fibrinolytic system associated with physical conditioning. Eur J Appl Physiol 1992:65:388-93

43 Gough SCL, Whitworth S, Rice PJS, et al. The effect of exercise and heart rate on fibrinolytic activity. Blood Coagul Fibrinolysis 1992;3:179-82.

44 Szymanski LM, Pate RR. Effect of exercise intensity, duration, and time of day on fibrinolytic activity in physically active men. Med Sci Sports Exerc 1994;26: $1102-8$

45 Handa K, Terao Y, Mori T, et al. Different coagulability and fibrinolytic activity during exercise depending on exercise intensities. Thromb Res 1992;66:613-16.

46 El-Sayed MS. Exercise intensity-related responses of fibrinolytic activity and vasopressin in man. Med Sci Sports Exerc 1990;22:494-500.

47 Bartsch P, Welsch B, Albert $M$, et al. Balanced activation of coagulation and fibrinolysis after a $2 \mathrm{~h}$ triathlon. Med Sci Sports Exerc 1995;27:1465-70.

48 Gonzales F, Manas M, Seiquer I, et al. Blood platelet function in healthy individuals of different ages. Effects of exercise and exercise conditioning. J Sports Med Phys Fitness 1996;36:1 12-16.

49 Siegel AJ, Stec JJ, Lipinska I, et al. Effect of marathon running on inflammatory and hemostatic markers. Am J Cardiol 2001;88:918-20.

50 Whitten CW, Greilich PE. Thromboelastography: past, present, and future. Anesthesiology 2000;92:1223-5. 